\title{
FINITE-ELEMENT METHODS AND MULTI-FIELD APPLICATIONS
}

\author{
MUHAMMAD ILYAS ${ }^{\circledR}$
}

(Received 22 February 2020; first published online 20 May 2020)

2010 Mathematics subject classification: primary 65N30; secondary 65N15.

Keywords and phrases: finite-element method, Poisson problem, Nitsche penalty method, gradient recovery method, elasticity problem, sixth order problem, ice shelf vibration problems.

Partial differential equations have a long and rich history of application in physical problems. Most of the partial differential equations arising from real life applications cannot be solved analytically. For these equations, one has to apply a numerical approximation technique. The finite-element method is a very popular technique to approximate solutions of partial differential equations. The finite-element method is based on rigorous mathematical foundations and convergence analysis, proving that it is a reliable approximation technique.

This thesis focuses on multi-field formulations for the Poisson problem, elasticity problem and sixth-order problem, where we use mixed formulations to get a better approximation of gradient, stress, pressure, vorticity, etc. In contrast to earlier approaches, we adopt a biorthogonal approach to obtain an efficient numerical scheme. We use a three-field and five-field formulation for the Poisson and elasticity problem, respectively, which allow us to use a biorthogonal system for efficient finite-element methods. The use of biorthogonal systems leads to a diagonal coupling matrix and, thus, all the auxiliary variables can be statically condensed out from the system. The auxiliary variables are gradient and Lagrange multipliers for the Poisson problem, whereas pressure, stress and strain are auxiliary variables for the elasticity problem. Hence we get a reduced system with only primal solution variables to solve. The sixthorder problem is also written as a three-field mixed formulation. However, we cannot use a biorthogonal system in this case. We also utilise the finite-element method to solve the vibration of the ice shelf and show that our approach works better than the traditional method. In this case, the finite-element method has the advantage in a water cavity with arbitrary shape, which enables us to solve more realistic models.

Thesis submitted to the University of Newcastle in December 2018; degree approved on 31 May 2019; supervisor Bishnu Lamichhane, associate supervisor Michael Meylan.

(c) 2020 Australian Mathematical Publishing Association Inc. 
For the Poisson problem, we develop three new approaches based on an abstract mixed formulation. The first formulation is the modification of a primal mixed formulation, in which we introduce a stabilisation term to satisfy well-posedness conditions. We formulate a new parametrised stabilisation term and show the optimal parameter can be found using the extended Céa lemma. On the second approach, we extend the parameterised mixed formulation with the Nitsche penalty method to weakly define the essential (Dirichlet) boundary conditions. The Nitsche formulation, combined with a biorthogonal system, allows us to preserve the optimal convergence and have efficient numerical computation. The third approach is the modification of a dual mixed formulation, where we use the Raviart-Thomas element to discretise the gradient. We construct a biorthogonal system locally on each element and condense out degrees of freedom related to the gradient and the Lagrange multiplier, to arrive at the system with only the primal solution variables. Some numerical examples are presented to evaluate and compare the three formulations. All three Poisson problem formulations are numerically efficient compared to the standard mixed formulation, due to the use of the biorthogonal system.

In the next part of the thesis, we are interested in the application of the boundary modification technique, which is widely used in the context of the mortar finiteelement method, to the gradient recovery approach. In this case, we construct a new gradient recovery method utilising both the biorthogonal system and the boundary modification technique. The key to the new formulation is the boundary modification technique, which preserves the convergence rates on the boundary patch shown by some numerical examples. The boundary modification technique is also used in the clamped boundary condition for the sixth-order problem to modify the Lagrange multiplier space so that it satisfies the required approximation property. We then use the constrained minimisation to cast our problems in a three-field mixed formulation as in the case of the biharmonic equation. Some numerical examples, utilising the linear and quadratic finite elements, are shown to agree with our theoretical convergence rates.

The elasticity problem is also one of the applications of the biorthogonal approach developed in this thesis. The combination of the standard $\mathrm{Hu}-\mathrm{Washizu}$ and the displacement-pressure approach leads us to the five-field formulation. As in the case of the Poisson problem, we use a biorthogonal system to get an efficient numerical method. Using such a biorthogonal system for the strain and stress, pressure and its Lagrange multiplier, the stress, strain, pressure and its Lagrange multiplier can be statically condensed out from the system just by inverting diagonal matrices.

The last problem considered in this thesis is the application of the finite-element method in the ice-shelf vibration problem. The problem can be seen as an elastic fluid-solid body interaction in a simplified setting. In this case, rather than elastic coupling, we use the Bernoulli-Euler beam assumption for the ice shelf and solve the fourth-order partial differential equation for the coupling problem. We show that our approach works well by using a few benchmark examples.

Some of this research has been published in [1-5]. 


\section{References}

[1] M. Ilyas and B. P. Lamichhane, 'A detailed mixed finite emelemnt method for the Poisson problem based on a three-field formulation', in: Proceedings of the 12th Biennial Engineering Mathematics and Applications Conference, EMAC-2015, ANZIAM J. 57 (2016), C177-C192.

[2] M. Ilyas, B. P. Lamichhane and M. H. Meylan, "A gradient recovery method based on an oblique projection and boundary modification', in: Proceedings of the 18th Biennial Computation Techniques and Applications Conference, CTAC-2016, ANZIAM J. 58 (2017), C34-C35.

[3] M. Ilyas and B. P. Lamichhane, 'A three-field formulation of the Poisson problem with Nitsche approach', in: Proceedings of the 13th Biennial Engineering Mathematics and Applications Conference, EMAC-2017, ANZIAM J. 59 (2018), C128-C142.

[4] J. Dromiou, M. Ilyas, B. P. Lamichhane and G. E. Wheeler, 'A mixed finite element method for a sixth-order elliptic problem', IMA J. Numer. Anal. 39(1) (2017), 374-397.

[5] M. Ilyas, M. H. Meylan, B. P. Lamichhane and L. G. Bennetts, 'Time-domain and modal response of ice shelves to wave forcing using the finite element method', J. Fluids Struct. 80 (2018), 113-131.

MUHAMMAD ILYAS, School of Mathematical and Physical Sciences, University of Newcastle, Callaghan, NSW 2308, Australia

e-mail: muhammad.ilyas@uon.edu.au 